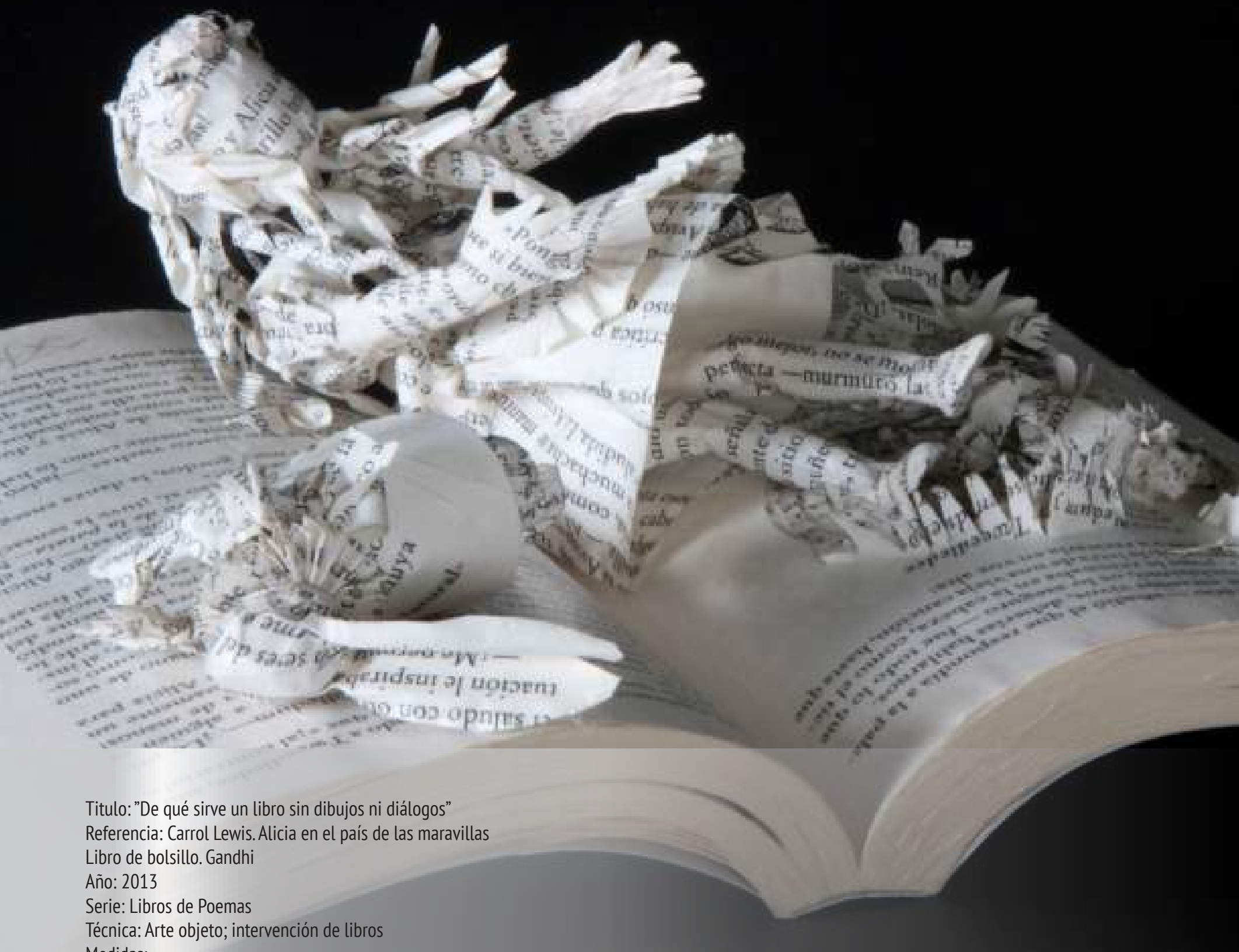

Medidas:

Foto: Pablo Cuéllar

ISSN 2007-8250/p.14 - p.27 


\title{
Actitudes, autopercepción de logro y contextualización de los aprendizajes en Física
}

\author{
Attitudes, self-perception of achievement \\ and contextualization of learning in Physic
}

Pedro Alonso Serna Sánchez

\section{Resumen}

El presente trabajo de investigación explora la acción vinculadora de las actitudes, percepciones y creencias de los estudiantes hacia la Física, sobre la autopercepción de logro en el desarrollo de competencias científicas y la contextualización de sus aprendizajes, a partir de una estrategia didáctica sustentada en actividades experimentales y en el uso de recursos multimedia. Los participantes fueron estudiantes de tercer semestre de bachillerato: un grupo control en el que no se realizó intervención y dos grupos experimentales, uno correspondiente al programa de bachillerato general y otro al bachillerato bilingüe progresivo, en los que se implementó la estrategia didáctica durante el semestre académico. El trabajo se concibió con un alcance explicativo, bajo una metodología cuantitativa con un diseño cuasiexperimental. La estrategia utilizada mejoró significativamente las actitudes y percepciones de los estudiantes hacia la Física, vinculando favorablemente a la autopercepción de logro y la contextualización de los aprendizajes en uno de los grupos donde se realizó la intervención, en el otro sólo incrementaron significativamente su nivel de contextualización y en el grupo control no presentó cambios significativos en ninguna de las tres variables consideradas. Se concluye que un cambio positivo en las actitudes de los estudiantes, vincula favorablemente la autopercepción de logro y la contextualización de los aprendizajes en la Física.

Palabras clave: actitudes, autopercepción, contextualización, aprendizajes, estrategia, didáctica.

\section{Abstract}

The present research explored the linking effect of students' attitudes, perceptions and beliefs toward Physics, about the self-perception of achievement in the development of scientific competences and the contextualization of their learning, based on a didactic strategy supported on experimental activities and in the use of multimedia resources. Participants were third semester high school students: a control group ( $\mathrm{N}=32)$ in which no intervention was performed and two experimental groups, one corresponding to the general baccalaureate program $(\mathrm{N}=32)$ and another to the progressive bilingual baccalaureate $(\mathrm{N}=27)$, in which the didactic strategy was implemented during the academic semester. The work was conceived with an explanatory scope, under a quantitative methodology with a quasi-experimental design. A pretest and postest were applied at the beginning and end of the academic semester in the three groups considered. The strategy used significantly improved students' attitudes and perceptions toward physics, favorably linking self-perception of achievement and the contextualization of learning in the progressive bilingual baccalaureate group. In the other experimental group, although students did not improve their attitudes, perceptions and beliefs, or self-perception of achievement, they significantly increased their level of contextualization. The control group did not present significant changes in any of the three variables considered. It is concluded that a positive change in students' attitudes, favorably links the self-perception of achievement and the contextualization of learning in Physics.

Keywords: attitudes, self-perception, contextualization, learning, strategy, didactics. 
Año 6 | No.11 | Enero-Junio 2018 Actitudes, autopercepción de logro y contextualización de los aprendizajes en Física

pesar de la importancia de la
Física como ciencia en el desa-
rrollo del hombre y de la sociedad, su enseñanza en el nivel medio superior ha presentado complejas problemáticas relacionadas con las actitudes iniciales desfavorables de los estudiantes hacia ella y con el propio proceso de enseñanza-aprendizaje, tal como lo demuestran numerosas investigaciones al respecto (Acevedo Díaz, 2007; Demirci, 2004; Pinochet y Rivera, 2014; Vázquez Alonso y Manassero Mas, 2008; Williams et al., 2003; Zwickl et al., 2013).

El enfoque tradicional se ha centrado en la enseñanza de conceptos teóri$\cos$ y ejemplos numéricos sin relación alguna con la realidad, sin dejar espacio al razonamiento analítico, crítico y reflexivo (Lara-Barragán Gómez, 2012; Lara-Barragán Gómez y Cerpa Cortés, 2014; Rioseco y Romero; 1997).

La creatividad del docente para plantear en forma contextualizada los temas a tratar, es fundamental para la implementación de entornos adecuados de aprendizaje, con el objetivo de mejorar las actitudes de los estudiantes hacia la Física y lograr un aprendizaje realmente significativo.

La realización de actividades experimentales y la utilización de simulaciones computacionales, han demostrado ser efectivas para lograr estos entornos de aprendizaje (Barbosa y Mora-Ley, 2010; Bouciguez y Santos; 2010; Fiscarellia et al., 2013; Marulanda y Gómez, 2006; Rao Aravind y Heard, 2010). Un cambio favorable en las actitudes de los estudiantes hacia la Física, podría

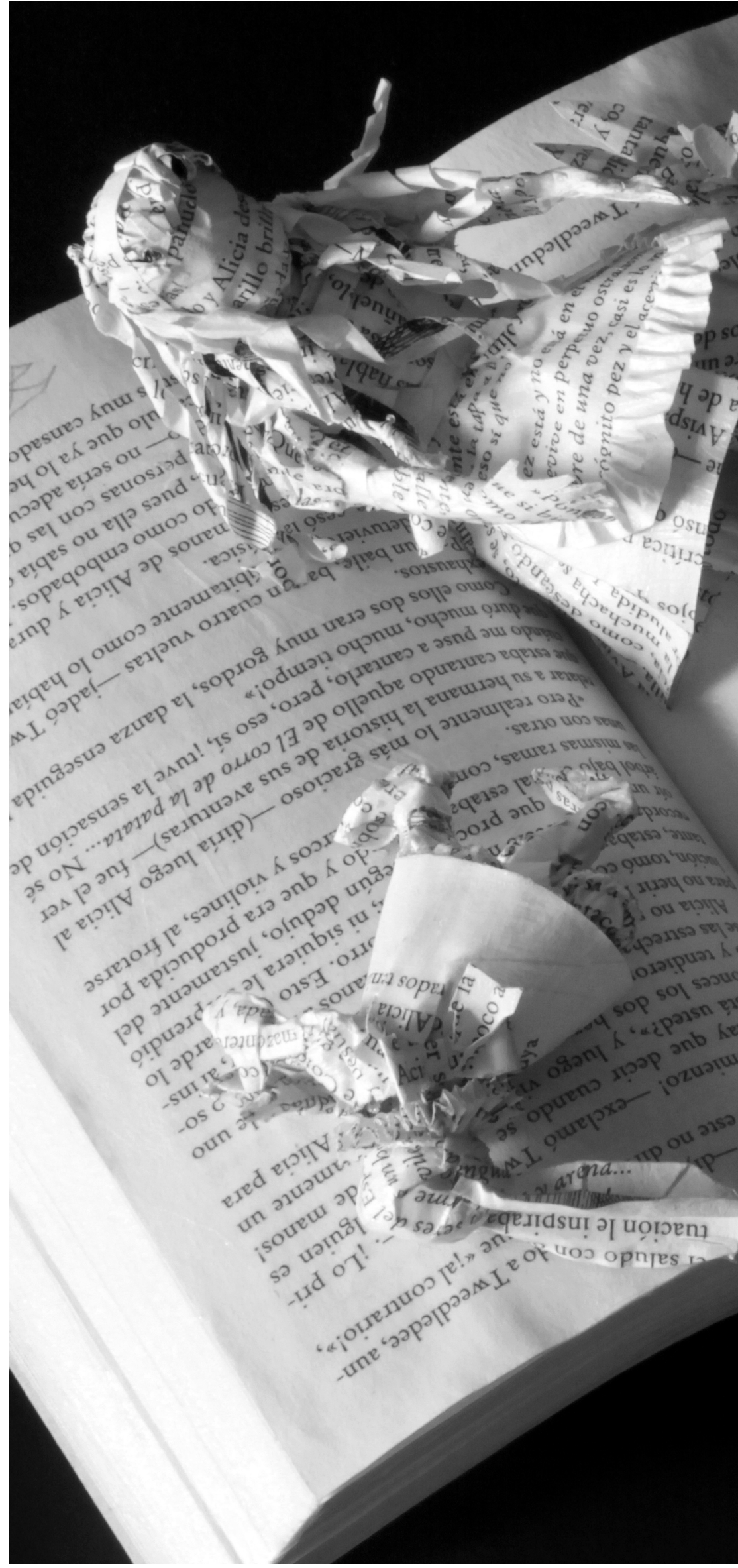


mejorar la autopercepción de logro y la contextualización de lo que se aprende.

Diversos estudios internacionales basados en la aplicación del TIMSS (Third International Mathematics and Science Study) y de PISA (Programa para la Evaluación Internacional de los alumnos de países de la OCDE) han mostrado resultados interesantes en relación a estas dos variables: en países en donde los estudiantes presentaban autoevaluaciones más altas, generalmente los resultados eran muy pobres en las pruebas objetivas y viceversa.

A su vez, los países con una alta autopercepción de logro de los estudiantes, presentaban un desempeño muy bajo en las pruebas (Shen y Pedulla, 2000; Shen y Talavera, 2003; Wilkins, 2004; Gutiérrez, 2008).

Estos resultados muestran lo complejo de la relación entre la autopercepción de logro de los estudiantes y el nivel de contextualización de sus aprendizajes.

El planteamiento del problema en el presente trabajo es el siguiente: ¿Qué relevancia tienen las actitudes, percepciones y creencias de los estudiantes hacia la Física sobre la relación entre la autopercepción de logro en el desarrollo de competencias científicas y la contextualización de los aprendizajes, a partir de una estrategia didáctica basada en actividades experimentales y en el uso de recursos multimedia?

El objetivo general de la presente investigación es explorar la relevancia que tienen las actitudes, percep- ciones y creencias de los estudiantes hacia la Física, sobre la relación entre la autopercepción de logro en el desarrollo de competencias científicas y la contextualización de los aprendizajes, a partir de la realización de actividades experimentales y el uso de recursos multimedia.

A continuación se exponen los objetivos específicos de la investigación: 1. Explorar y describir la relevancia de la estrategia didáctica utilizada por el docente, basada en actividades experimentales y en el uso de recursos multimedia, sobre: (a) las actitudes, percepciones y creencias de los estudiantes hacia la Física, (b) la autopercepción de logro en el desarrollo de competencias científicas y (c) el nivel de contextualización de sus aprendizajes.

2. Caracterizar las relaciones entre las actitudes, percepciones y creencias hacia la Física, la autopercepción de logro en el desarrollo de las competencias científicas de los estudiantes y el nivel de contextualización de sus aprendizajes.

La hipótesis planteada en el presente trabajo es la siguiente: Las actitudes, percepciones y creencias positivas de los estudiantes hacia la Física.

Vinculan en forma favorable y significativa a la autopercepción de logro en el desarrollo de competencias científicas y a la contextualización de los aprendizajes, a partir de una estrategia didáctica basada en actividades experimentales y en el uso de recursos multimedia.

En general, la ciencia que se enseña en las escuelas, y particularmente

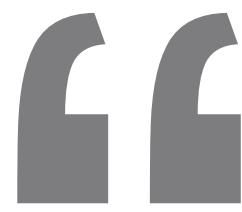

El enfoque

tradicional se

ha centrado en

la enseñanza de conceptos teóricos y ejemplos numéricos sin relación alguna con la realidad, sin dejar espacio al razonamiento analítico, crítico y reflexivo". 


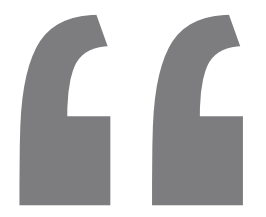

\section{El autoconcepto}

en ciencias de una persona, se refiere a las percepciones o creencias sobre

sus propias

habilidades para

desempeñarse

bien en el campo de las ciencias". la Física, suele considerarse por los estudiantes como aburrida, impersonal, desconectada de sus propios intereses e irrelevante para la sociedad en general, lo cual origina que la mayoría de ellos se formen una imagen distorsionada de ella.

Diversos estudios muestran resultados desalentadores en relación al desinterés que muestran los jóvenes, particularmente en el bachillerato, por seguir una carrera científica. Esto abre el debate acerca de la forma en la que se enseña la Física y las ciencias hoy en día.

Los estudiantes visualizan a la Física como una asignatura difícil, llena de conceptos teóricos, de formulismos matemáticos y sin ninguna vinculación con el mundo real.

Esto es consecuencia del modo en que se ha abordado la materia por parte de los docentes, de una forma totalmente descontextualizada y desconectada de su experiencia diaria, de tal manera que no le permite al estudiante darle sentido y significado a lo que aprende.

La función del docente como facilitador de los aprendizajes en los nuevos contextos educativos, le obliga a buscar estrategias de enseñanza aprendizaje innovadoras que generen interés en los estudiantes por aprender los principios y fundamentos de cada unidad de aprendizaje.

Por tal motivo, en el presente trabajo de investigación se pretende implementar actividades relacionadas con la experimentación y el uso de los recursos informáticos, con el fin de motivar y despertar el interés de los estudiantes y cambiar favorablemente sus actitudes, percepciones y creencias iniciales hacia la Física.

Así mismo, al cambiar sus actitudes, percepciones y creencias, hace posible una mejora en la autopercepción de logro en el desarrollo de sus propias competencias, creando las condiciones necesarias para lograr una adecuada contextualización de los aprendizajes, asignándole un significado a los conocimientos adquiridos.

Actualmente la enseñanza de la Física a nivel de bachillerato puede ser calificada como tradicional, es decir, está sustentada en la transmisión de conocimientos y en la figura del docente como elemento central.

Hace falta un cambio fundamental que se refleje en un aprendizaje más constructivo, que ayude al estudiante a aprender de forma más significativa y colaborativa. Es de los estudiantes y mejorar sus actitudes iniciales negativas, en ambientes que promuevan un enfoque constructivista y que permitan el logro de un aprendizaje realmente significativo y contextualizado.

Por otro lado, el autoconcepto en ciencias de una persona, se refiere a las percepciones 0 creencias sobre sus propias habilidades para desempeñarse bien en el campo de las ciencias (Wilkins, 2004).

Muy relacionado con este concepto, la autopercepción de logro en el ámbito académico, se puede definir como las percepciones 0 creencias que los estudiantes tienen en relación al dominio que han desarrollado sobre 


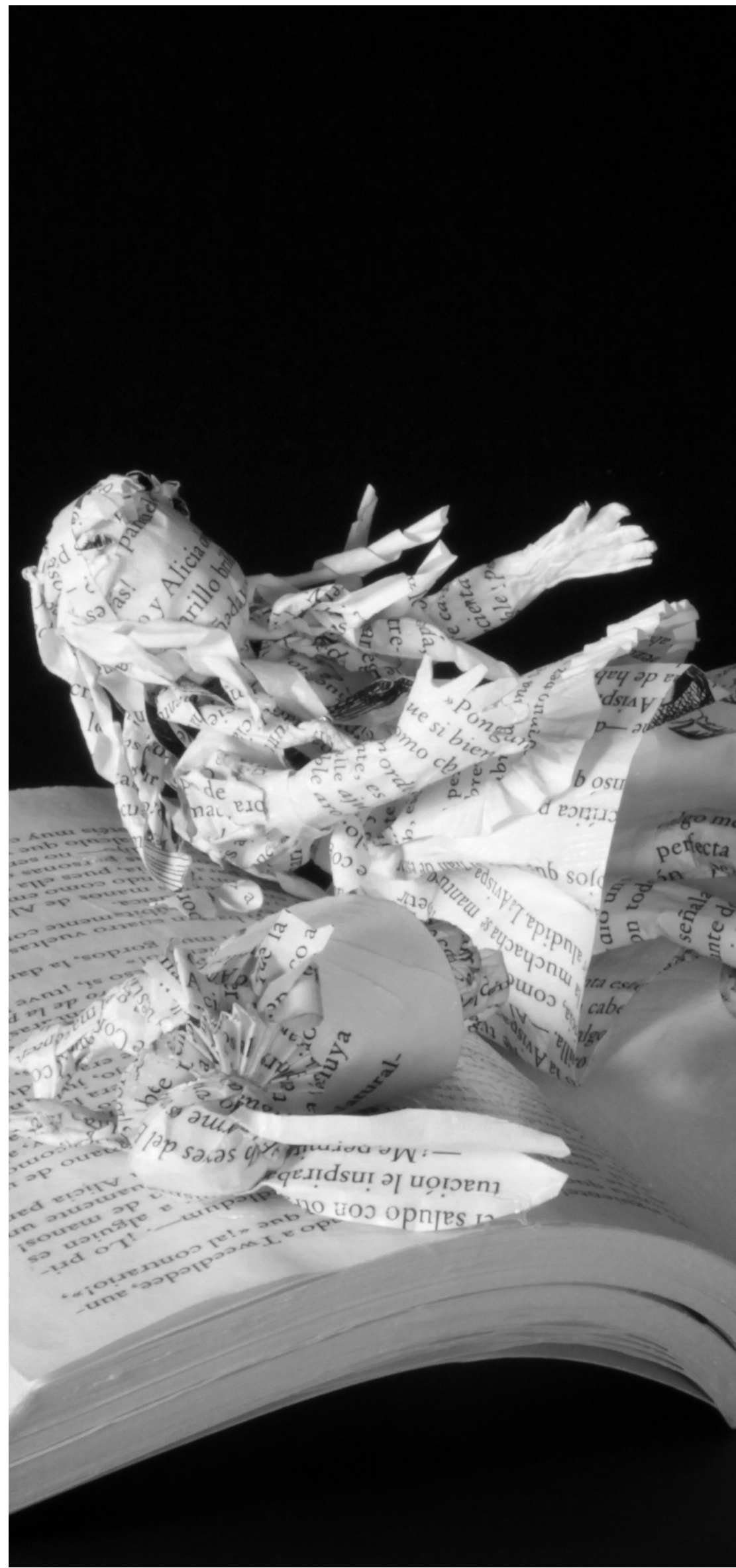

las competencias requeridas en cierta área del conocimiento.

A su vez, la enseñanza y el aprendizaje contextualizado es una concepción del proceso educativo que ayuda a los profesores a relacionar los contenidos teóricos de las unidades de aprendizaje con situaciones del mundo real y motivan a los estudiantes a realizar conexiones entre los conocimientos adquiridos y sus aplicaciones en sus propias vidas (Berns y Erickson, 2001).

La implementación de adecuados contextos, a partir de estrategias didácticas relevantes, es fundamental para incrementar la motivación y el interés de los estudiantes hacia el aprendizaje (Shiu-sing, 2005; Whitelegg y Parry, 1999).

Hasta ahora, pocos trabajos de investigación se han realizado en el nivel medio superior, que aborden la relación entre las actitudes, percepciones y creencias de los estudiantes hacia la Física.

La autopercepción de logro en el desarrollo de competencias científicas y el nivel de contextualización de sus aprendizajes, a partir de una estrategia didáctica que incluya actividades relevantes y significativas.

La presente investigación se realizó en la Escuela Preparatoria 24 "Dr. Alfredo Piñeyro López" de la UANL, ubicada en el municipio de Anáhuac, N.L. Se concibió con un alcance explicativo, bajo una metodología cuantitativa con un diseño considerado un grupo control y dos grupos experimentales, con una población escolar total de 91 estudiantes. 


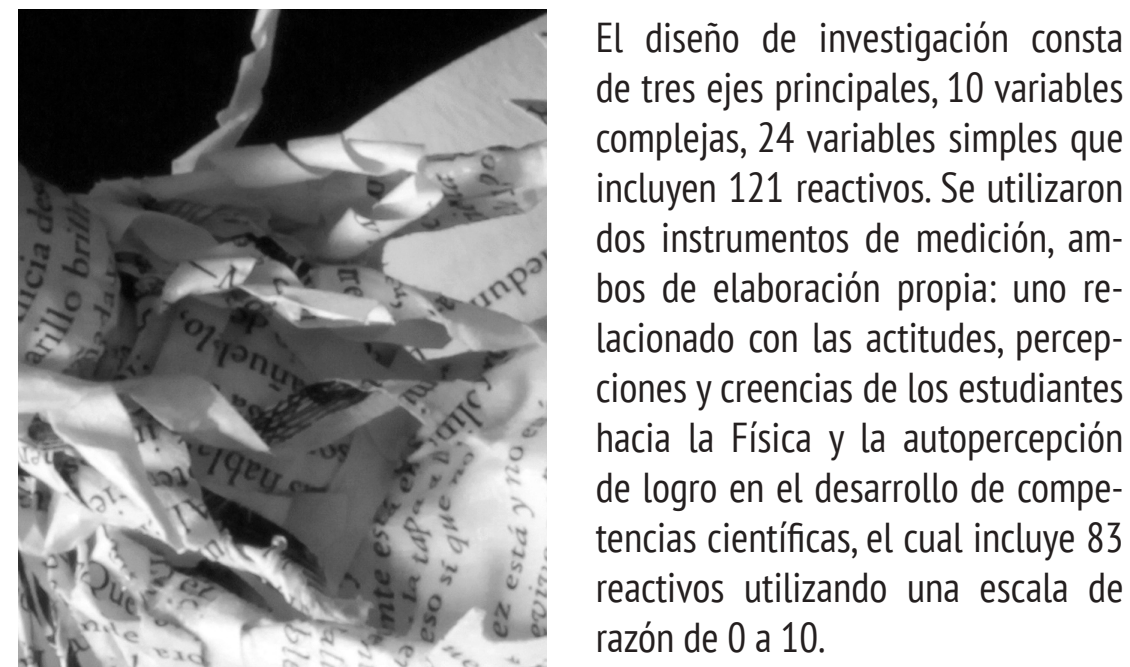

Otro correspondiente a la evaluación de la contextualización de los aprendizajes, el cual consta de 38 reactivos de tipo nominal dicotómico, en la tabla 1 podemos observar los ejes, variables complejas, variables simples y niveles de medición de los instrumentos utilizados.

Ambos instrumentos fueron puestos a consideración de jueces especialistas en el área, las aportaciones y recomendaciones fueron consideradas en el diseño final del instrumento.

Con respecto al análisis de fiabilidad y validez del instrumento diseñado para medir las actitudes, percepciones y creencias de los estudiantes hacia la Física y la autopercepción de logro en el desarrollo de competencias científicas, se realizó a partir de una prueba piloto que se aplicó a 37 estudiantes de cuarto semestre de la escuela que no participaron en la propuesta de intervención.

El nivel de contextualización de los aprendizajes logrados por los estudiantes, lográndose un valor del Coeficiente alfa de Cronbach de 0.70 para todo el instrumento, diversos autores establecen que por tratarse de reac- tivos de tipo nominal dicotómico que evalúan de cierta forma conocimientos, no son aplicables los coeficientes alfa de Cronbach y las correlaciones promedio ítem-total (Campo-Arias y Oviedo, 2008; Streiner, 2003).

Con respecto al análisis de fiabilidad de los instrumentos finales, se realizó a partir de los datos obtenidos de la aplicación del pretest, en los dos grupos experimentales y en el grupo control considerados, mediante la determinación del coeficiente alfa de Cronbach (ver tabla 2), los datos recabados se analizaron a través del paquete estadístico SPSS 19.

Al inicio del curso se aplicaron dos pretest relacionados con los dos instrumentos de medición utilizados; posteriormente se realizó la intervención experimental y, al final del curso, se aplicaron dos postest.

La estrategia didáctica consistió en la realización de 32 diversas actividades experimentales de tipo cualitativo (ver tabla 3), y la utilización de 26 recursos multimedia como simulaciones computacionales (ver tabla 4) y 20 videos cortos (ver tabla 5), todos estos recursos didácticos relacionados con los temas que se abordaron durante el curso. La implementación de estas actividades fue en diferentes momentos al tratarse un tema en particular. Se realizaron análisis estadísticos descriptivos, comparativos, correlacionales e integracionales.

Al comparar los puntajes totales de los conjuntos de variables de ambos instrumentos, antes y después de la intervención, los alumnos del grupo experimental correspondiente 
Tabla 1

Ejes, variables complejas, variables simples y niveles de medición de ambos instrumentos utilizados

Instrumento 1: Actitudes, percepciones y creencias hacia la Física y autopercepción de logro en el desarrollo de competencias científicas

\begin{tabular}{|c|c|c|c|}
\hline Eje & Variable Compleja & Variable Simple & Medición \\
\hline \multirow{3}{*}{$\begin{array}{l}\text { Actitudes, percepciones y } \\
\text { creencias hacia la Física. }\end{array}$} & $\begin{array}{c}\text { Actitudes hacia la } \\
\text { Física }\end{array}$ & $\begin{array}{c}\text { Atracción hacia la Física } \\
\text { Curiosidad natural } \\
\text { Gusto por la experimentación }\end{array}$ & $\begin{array}{l}\text { Razón } \\
\text { Razón } \\
\text { Razón }\end{array}$ \\
\hline & $\begin{array}{c}\text { Percepciones hacia la } \\
\text { Física }\end{array}$ & $\begin{array}{l}\text { Concepto de la Física como } \\
\text { una ciencia muy compleja } \\
\text { Noción de la Física como una } \\
\text { ciencia descontextualizada }\end{array}$ & $\begin{array}{l}\text { Razón } \\
\text { Razón }\end{array}$ \\
\hline & $\begin{array}{c}\text { Creencias hacia la } \\
\text { Física }\end{array}$ & $\begin{array}{c}\text { Alfabetización científica } \\
\text { Relación CTSS (Ciencia-Tecnología- } \\
\text { Sociedad-Sustentabilidad) }\end{array}$ & $\begin{array}{l}\text { Razón } \\
\text { Razón }\end{array}$ \\
\hline \multirow{4}{*}{$\begin{array}{l}\text { Autopercepción de logro } \\
\text { en el desarrollo de } \\
\text { competencias científicas }\end{array}$} & $\begin{array}{l}\text { Contexto de } \\
\text { aplicación }\end{array}$ & $\begin{array}{l}\text { Contexto personal } \\
\text { Contexto social } \\
\text { Contexto global } \\
\text { Contexto histórico }\end{array}$ & $\begin{array}{l}\text { Razón } \\
\text { Razón } \\
\text { Razón } \\
\text { Razón }\end{array}$ \\
\hline & Destrezas científicas & $\begin{array}{l}\text { Identificar cuestiones científicas } \\
\text { Explicar científicamente fenómenos } \\
\text { Tomar decisiones sustentadas } \\
\text { científicamente } \\
\end{array}$ & $\begin{array}{l}\text { Razón } \\
\text { Razón } \\
\text { Razón }\end{array}$ \\
\hline & $\begin{array}{l}\text { Conceptos } \\
\text { científicos }\end{array}$ & $\begin{array}{l}\text { Comprensión de conceptos científicos } \\
\text { Aplicación de conceptos científicos }\end{array}$ & $\begin{array}{l}\text { Razón } \\
\text { Razón }\end{array}$ \\
\hline & $\begin{array}{c}\text { Actitudes hacia la } \\
\text { ciencia }\end{array}$ & $\begin{array}{l}\text { Interés por contenidos científicos } \\
\text { Actitudes hacia los resultados } \\
\text { obtenidos por la ciencia } \\
\text { Actitudes hacia los científicos } \\
\text { y su trabajo }\end{array}$ & $\begin{array}{l}\text { Razón } \\
\text { Razón }\end{array}$ \\
\hline
\end{tabular}

\begin{tabular}{|c|c|c|c|}
\hline \multicolumn{4}{|c|}{ Instrumento 2: Contextualización de los aprendizajes } \\
\hline \multirow{3}{*}{$\begin{array}{c}\text { Contextualización de los } \\
\text { aprendizajes }\end{array}$} & $\begin{array}{l}\text { Comprensión de } \\
\text { fenómenos naturales }\end{array}$ & $\begin{array}{c}\text { Fundamentación teórica del fenómeno } \\
\text { Explicación del fenómeno }\end{array}$ & $\begin{array}{l}\text { Nominal } \\
\text { Nominal }\end{array}$ \\
\hline & $\begin{array}{c}\text { Aplicación en la vida } \\
\text { real }\end{array}$ & $\begin{array}{c}\text { Transferencia del conocimiento a } \\
\text { contextos reales }\end{array}$ & Nominal \\
\hline & Enfoque CTSS & $\begin{array}{l}\text { Impacto de la ciencia en la sociedad } \\
\text { Participación responsable en decisiones }\end{array}$ & $\begin{array}{l}\text { Nominal } \\
\text { Nominal }\end{array}$ \\
\hline
\end{tabular}


Análisis de fiabilidad del instrumento:

Actitudes, percepciones y creencias hacia la Física y autopercepción de logro en el desarrollo de competencias cientíicas

\begin{tabular}{lccc}
\hline \multicolumn{1}{c}{ Conjunto } & Elementos & $\begin{array}{c}\text { Alfa de } \\
\text { Cronbach }\end{array}$ & $\begin{array}{c}\text { Correlación } \\
\text { promedio ítem-total }\end{array}$ \\
\hline Instrumento completo & 83 & 0.968 & 0.274 \\
\hline Atracción hacia la Física & 5 & 0.876 & 0.589 \\
Curiosidad natural & 4 & 0.823 & 0.544 \\
Gusto por la experimentación & 4 & 0.872 & 0.651 \\
Concepto de la Física como una ciencia muy compleja & 6 & 0.877 & 0.543 \\
Noción de la Física como una ciencia descontextualizada & 4 & 0.897 & 0.688 \\
Alfabetización científica & 4 & 0.766 & 0.450 \\
Relación CTSS (Ciencia-Tecnología-Sociedad-Sustentabilidad) & 4 & 0.859 & 0.604 \\
\hline Contexto personal & 4 & 0.766 & 0.446 \\
Contexto social & 4 & 0.793 & 0.493 \\
Contexto global & 4 & 0.890 & 0.678 \\
Contexto histórico & 4 & 0.923 & 0.756 \\
Identificar cuestiones científicas & 4 & 0.819 & 0.534 \\
Explicar científicamente fenómenos & 6 & 0.907 & 0.618 \\
Tomar decisiones sustentadas científicamente & 4 & 0.811 & 0.522 \\
Comprensión de conceptos científicos & 5 & 0.906 & 0.662 \\
Aplicación de conceptos científicos & 5 & 0.898 & 0.643 \\
Interés por contenidos científicos & 4 & 0.781 & 0.474 \\
Actitudes hacia los resultados obtenidos por la ciencia & 4 & 0.889 & 0.669 \\
Actitudes hacia los científicos y su trabajo & 4 & 0.860 & 0.616 \\
\hline
\end{tabular}

Tabla 3

Actividades experimentales de la propuesta de intervención

\begin{tabular}{ccc}
\hline Etapa & Actividad experimental & Tiempo \\
Etapa 1. & Aplicación de vectores \\
Vectores & Vectorímetro \\
(1era. Parte) & Más vale maña que fuerza & 50 Minutos \\
& El hilo que se rompe por dos lugares & \\
\hline
\end{tabular}

\section{Experimento sobre gravedad y equilibrio}

Lata equilibrista

Otro equilibrio con un tenedor, una cuchara, un palillo y fuego

Figuras equilibristas

Centro de gravedad de nuestro cuerpo

Etapa 1. Primera Ley de Newton. Inercia "La moneda desobediente"

Dinámica

(2da. Parte)

Inercia, fricción y fuerza "Demoliendo la torre"

100 Minutos

Segunda Ley de Newton

Carrera de globos

Aerodeslizador casero

Coeficiente de fricción estático 
Etapa

Etapa 2

Gravitación
Actividad

Experimental
Tiempo

\section{Ingravidez aparente}

Peso y fuerza centrípeta

Fuerza centrípeta en un cubo con agua

Fuerza centrípeta

50 Minutos

Concepto de gravedad de Einstein

Curvatura del espacio-tiempo

Simulando agujeros negros

Paradoja del plano inclinado

Péndulo interrumpido

Trabajo mecánico

50 Minutos

Trabajo, energía

y potencia

La montaña rusa

Cambio de energía

Experimento sobre transformación de energía

Etapa 4

Impulso y cantidad

de movimiento y cantidad de movimiento

Cañón de Gauss casero

Péndulo de Newton

Choques elásticos e inelásticos

Impulso y cantidad de movimiento

Tabla 4

Actividades con laboratorios y simulaciones virtuales de la propuesta de intervención

\begin{tabular}{|c|c|c|}
\hline Etapa & $\begin{array}{c}\text { Laboratorio o } \\
\text { simulación virtual }\end{array}$ & Tiempo \\
\hline $\begin{array}{c}\text { Etapa } 1 \\
\text { Vectores } \\
\text { (1era. Parte) }\end{array}$ & $\begin{array}{l}\text { Fundamentos de fuerzas y movimiento. PhET } \\
\text { Adición de vectores. PhET } \\
\text { Suma de vectores. Método del paralelogramo. Educaplus } \\
\text { Suma de dos vectores. Educaplus } \\
\text { Suma de componentes de un vector. David M. Harrison }\end{array}$ & 25 minutos \\
\hline $\begin{array}{c}\text { Etapa } 1 \\
\text { Dinámica } \\
\text { (2da. Parte) }\end{array}$ & $\begin{array}{c}\text { Segunda Ley de Newton (Segundo principio) } \\
\text { Aceleración en un plano inclinado } \\
\text { Descomposición del peso de un cuerpo en un plano } \\
\text { inclinado. Educaplus. } \\
\text { Dinámica de un móvil en un plano inclinado con } \\
\text { rozamiento. Educaplus } \\
\text { Fuerza de Fricción. Universidad de Colorado PhET } \\
\text { Fricción estática y cinética. FisQuiWeb }\end{array}$ & 25 minutos \\
\hline $\begin{array}{c}\text { Etapa } 2 \\
\text { Gravitación }\end{array}$ & $\begin{array}{c}\text { Fuerzas en el giro de los planetas. Educaplus } \\
\text { Laboratorio fuerza de gravedad. PhET } \\
\text { Interacción gravitatoria. Educaplus } \\
\text { Mi Sistema Solar. PhET } \\
\text { Lanzamiento horizontal de un martillo. Educaplus } \\
\text { Ley dela Gravitación Universal. Salvador Hurtado Fernández }\end{array}$ & 25 minutos \\
\hline
\end{tabular}


Actividades con laboratorios y simulaciones virtuales de la propuesta de intervención.

\begin{tabular}{ccc}
\hline Etapa & $\begin{array}{c}\text { Laboratorio o } \\
\text { simulación virtual }\end{array}$ & Tiempo \\
Etapa 3 & Energía potencial gravitacional. Educaplus & \\
Prabajo, energía & Péndulo simple \\
y potencia & Conservación de la energía en el péndulo. Educaplus & 25 minutos \\
& Planta hidroeléctrica & \\
& Pista de patinar “energía”. PhET & \\
Etapa 4 & Choque inelástico. Educaplus. & \\
Impulso y cantidad & Choque elástico. Educaplus & 25 minutos \\
de movimiento & Conservación de la cantidad de movimiento & \\
\hline
\end{tabular}

Tabla 5

Videos cortos utilizados durante la propuesta de intervención

\begin{tabular}{|c|c|c|}
\hline Etapa & Nombre del video corto & Tiempo \\
\hline $\begin{array}{c}\text { Etapa } 1 \\
\text { Vectores } \\
\text { (1era. Parte) }\end{array}$ & $\begin{array}{c}\text { Vectores, definiciones básicas } \\
\text { Suma de vectores. Método del polígono }\end{array}$ & 25 minutos \\
\hline $\begin{array}{l}\text { Etapa } 1 \\
\text { Dinámica } \\
\text { (2da. Parte) }\end{array}$ & $\begin{array}{c}\text { El Universo, la fuerza y el movimiento } \\
\text { Las tres leyes de Newton (Leyes del movimiento) } \\
\text { Las leyes del movimiento de Newton }\end{array}$ & 25 minutos \\
\hline $\begin{array}{l}\text { Etapa } 2 \\
\text { Gravitación }\end{array}$ & $\begin{array}{c}\text { Isaac Newton y la gravedad } \\
\text { Sencilla y gráfica explicación de la gravedad } \\
\text { Curvatura del espacio-tiempo } \\
\text { Leyes de Kepler } \\
\text { Avances tecnológicos. Satélites artificiales } \\
\text { Vista nocturna del planeta Tierra } \\
\text { Ley de Gravitación }\end{array}$ & 25 minutos \\
\hline $\begin{array}{l}\text { Etapa } 3 \\
\text { Trabajo, energía } \\
\text { y potencia }\end{array}$ & $\begin{array}{c}\text { Trabajo y energía } \\
\text { Trabajo, energía y movimiento } \\
\text { Energía mecánica } \\
\text { Potencia } \\
\text { Conservación de la energía mecánica }\end{array}$ & 25 minutos \\
\hline $\begin{array}{l}\text { Etapa } 4 \\
\text { Impulso y cantidad } \\
\text { de movimiento }\end{array}$ & $\begin{array}{c}\text { Choques } \\
\text { Energía. Walter Lewin } \\
\text { Principio de conservación de la cantidad de movimiento } \\
\text { Impulso y momento }\end{array}$ & 25 minutos \\
\hline
\end{tabular}


al programa de bachillerato bilingüe progresivo, mejoraron significativamente $(p \leqslant 0.05)$ sus actitudes y percepciones hacia la Física e incrementaron su autopercepción de logro, en relación a los alumnos del grupo experimental correspondiente al bachillerato general y a los del grupo control, también pertenecientes al programa de bachillerato general.

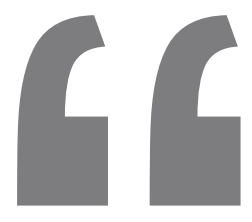

\section{La autopercepción} de logro en el desarrollo de competencias científicas

y el nivel de contextualización de sus aprendizajes".
A su vez, los alumnos de ambos grupos experimentales contextualizaron de mejor manera los aprendizajes, en relación al grupo control.

Se encontraron correlaciones positivas y significativas $(p \leqslant 0.05)$ entre las actitudes con la autopercepción de logro y con la contextualización de los aprendizajes de los estudiantes, así como entre estas dos últimas variables.

Para los tres grupos de estudio se procesaron modelos de regresión lineal simple con los datos del postest, considerando a las actitudes hacia la Física como variable predictora y a la autopercepción de logro como variable dependiente, con coeficientes de $\mathrm{R}=0.817$ para el grupo control, $\mathrm{R}=$ 0.741 para el grupo experimental uno y $R=0.713$ para el grupo experimental dos, aunque para éste último grupo, la variable predictora fue creencias y no actitudes hacia la Física.

En el Análisis de Componentes Principales (ACP) de los puntajes totales de los siete conjuntos correspondientes al instrumento Actitudes, percepciones y creencias hacia la Física y autopercepción de logro en el desarrollo de competencias científicas de los tres grupos en conjunto, antes y después de la intervención, se utilizó la medida de Káiser-Meyer-Olkin (KMO) para determinar el grado de adecuación de la matriz de correlaciones para la factorización, la Prueba de Esfericidad de Bartlett para determinar la dependencia entre las variables consideradas y el método de rotación oblicuo de Oblimin directo para la extracción de los factores.
Como resultado, se obtuvo una estructura factorial de 17 componentes y 79 reactivos en el pretest, en lugar de las 19 componentes y 83 reactivos del instrumento original.

Para los datos del postest, el ACP generó una estructura factorial de 17 componentes, pero manteniendo los 83 reactivos del instrumento original. El Análisis Factorial Confirmatorio (AFC) utilizando el método de Mínimos Cuadrados Generalizados, realizado a los mismos conjuntos de datos, permitió confirmar las componentes determinadas por el ACP.

A partir de los resultados de este trabajo de investigación, se concluye que las actividades experimentales y la utilización de recursos multimedia como estrategia didáctica en la enseñanza de la Física genera un cambio positivo y significativo en las actitudes y percepciones de los estudiantes de alto rendimiento hacia la Física; científicas en los alumnos de alto rendimiento y mejora notablemente el nivel de contextualización de los aprendizajes, tanto en alumnos de bachillerato general como en alumnos de bachillerato bilingüe progresivo, siendo mayor en éstos últimos.

En los estudiantes de alto rendimiento se cumplió el paradigma propuesto, ya que las actitudes y percepciones de los estudiantes hacia la Física vincularon favorablemente a la autopercepción de logro y a la contextualización de los aprendizajes. Este paradigma fue validado por pruebas estadísticas comparativas, correlacionales, modelos de regresión lineal simple, ACP y AFC. 


\section{Referencias}

Acevedo Díaz, J. A. (2007). Las actitudes relacionadas con la ciencia y la tecnología en el estudio PISA 2006. Revista Eureka sobre Enseñanza y Divulgación de las Ciencias, 4(3).

Barbosa, L. H. y Mora-Ley, C. (2010). Los experimentos discrepantes como una herramienta pedagógica en el aprendizaje de la Física. (Español). Discrepant experiments as a pedagogical tool in learning physics. (English), 42(1).

Berns, R. G., \& Erickson, P. M. (2001). Contextual Teaching and Learning: Preparing Students for the New Economy. The Highlight Zone: Research@ Work No. 5.

Bouciguez, M. J. y Santos, G. (2010). Applets en la enseñanza de la Física: un análisis de las características tecnológicas y disciplinares. Applets in Teaching Physics an Analysis of the Technologic and Disciplinary Characteristics (English) (Español) 7(1).

Demirci, N. (2004). Students' Attitudes toward Introductory Physics Course. Hacettepe University Journal of Education, 26.

Fiscarellia, S. H., Bizelli, M. H., \& Fiscarelli, P. E. (2013). Interactive simulations to physics teaching: a case study in Brazilian high school. International Journal of Learning and Teaching, 5(1).

Gutiérrez, A. (2008). La evaluación de las competencias científicas en PISA: perfiles en los estudiantes iberoamericanos. Alambique Didáctica de las Ciencias Experimentales, 57.

Lara-Barragán Gómez, A. y Cerpa Cortés, G. (2014). Enseñanza de la Física y desarrollo del Pensamiento Crítico. Lat. Am.J. Phys. Educ. Vol, 8(1).

Marulanda, J. I. y Gómez, L. A. (2006). Experimentos en el aula de clase para la enseñanza de la Física (Español) Revista Colombiana de Física, 38(2).

Pinochet, J.y Rivera, M. (2014).Adaptación y validación del Scale of Attitudes Towards Physics (SAP) en una muestra de estudiantes chilenos de ingeniería. Lat. Am. J. Phys. Educ. Vol, 8(1).

Rioseco, M. y Romero, R. (1997). La contextualización de la enseñanza como elemento facilitador del aprendizaje significativo. Actas Encuentro Internacional sobre el Aprendizaje Significativo.

Shen, C., \& Pedulla, J. J. (2000). The relationship between students' achievement and their self-perception of competence and rigour of mathematics and science: $A$ cross-national analysis. Assessment in Education: Principles, Policy \& Practice, 7(2).

Shen, C., \& Talavera, 0. (2003). The Effects of Self-perception on Students' Mathematics and Science Achievement in 38 Countries Based on TIMSS 1999 Data. Retrieved May, 25, 2010.

Shiu-sing, T. (2005). Some reflections on the design of contextual learning and teaching materials. Retrieved from Contextual Physics in Ocean Park http://resources. emb. gov. hk/ cphysics.

Vázquez Alonso, Á.,y Manassero Mas, M.A. (2008). El declive de las actitudes hacia la ciencia de los estudiantes: un indicador inquietante para la educación científica. Revista Eureka sobre Enseñanza y Divulgación de las ciencias, 5(3).

Whitelegg, E., \& Parry, M. (1999). Real-life contexts for learning physics: meanings, issues and practice. Physics Education, 34(2).

Wilkins, J. L. (2004). Mathematics and science self-concept: An international investigation. The Journal of Experimental Education, 72(4)0.

Williams, C., Stanisstreet, M., Spall, K., Boyes, E., \& Dickson, D. (2003). Why aren't secondary students interested in physics? Physics Education, 38(4), 324.

Zwickl, B. M., Finkelstein, N., \& Lewandowski, H.J. (2013). Development and validation of the Colorado Learning Attitudes about Science Survey for experimental physics. AIP Conference Proceedings, 1513(1), 442-445.

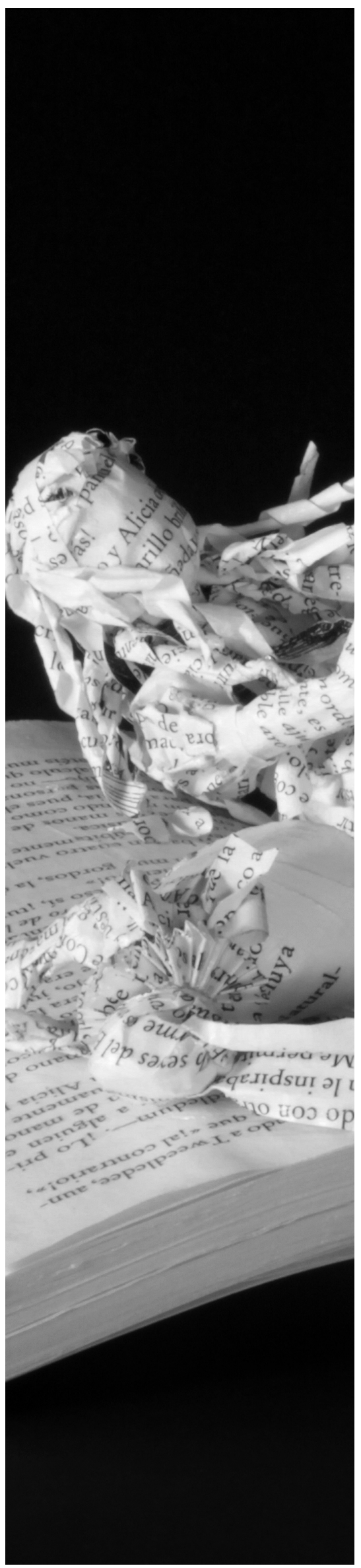



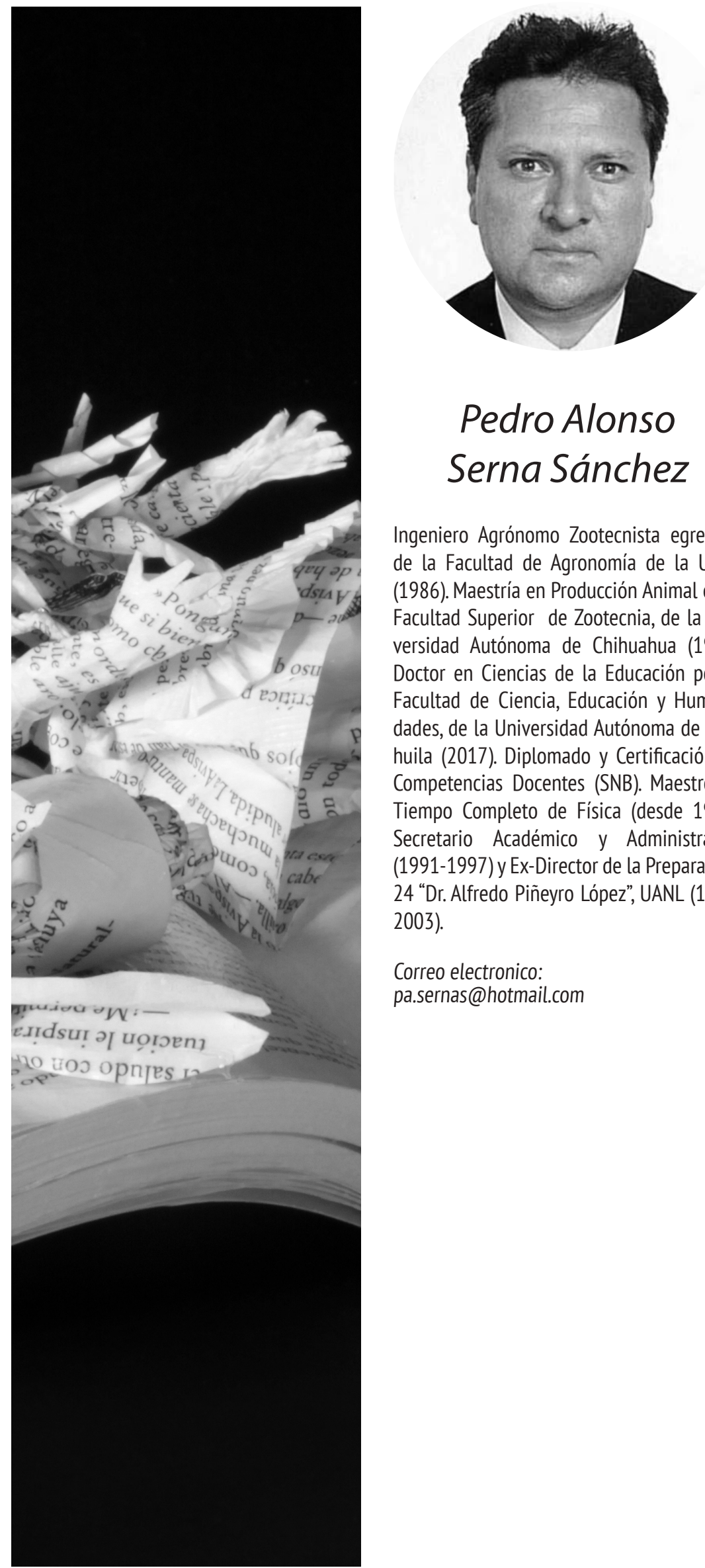

Ingeniero Agrónomo Zootecnista egresado de la Facultad de Agronomía de la UANL (1986). Maestría en Producción Animal en la Facultad Superior de Zootecnia, de la Universidad Autónoma de Chihuahua (1993). Doctor en Ciencias de la Educación por la Facultad de Ciencia, Educación y Humanidades, de la Universidad Autónoma de Coahuila (2017). Diplomado y Certificación en Competencias Docentes (SNB). Maestro de Tiempo Completo de Física (desde 1988), Secretario Académico y Administrativo (1991-1997) y Ex-Director de la Preparatoria 24 “Dr. Alfredo Piñeyro López”, UANL (19972003).

Correo electronico:

pa.sernas@hotmail.com

Recibido: 30/08/2017

Aceptado: 20/11/2017 